$\xi=-$ 圆

\title{
Performance Evaluation of G2T FSO Link Under Various Weather Conditions
}

\author{
Wafi A. Mabrouk ${ }^{1 *}$, M.F.L Abdullah ${ }^{2}$, M.S.M Gismalla ${ }^{3}$ \\ ${ }^{1,2,3}$ Faculty of Electrical \& Electronic Engineering, University Tun Hussien Onn Malaysia (UTHM), Parit Raja, Batu Pahat, Johor, \\ Malaysia. \\ *Corresponding author E-mail: wafi.mabrouk@gmail.com
}

\begin{abstract}
FSO technology has attracted a lot of popularity for a variety of applied telecommunication fields. It presents a wide range of advantages that place it in the frontier of high data rates applications, last mile problem and bottleneck issues. It has been preferred for its ease of deployment without fiber cables, no extra tariff fees, cost-effectiveness, and efficiency. FSO excels in performance when compared to contemporary RF technology. On the other hand, there is an increased demand for alternative rail communications solutions. In order to deliver a safer, reliable and fast internet access. In this paper, performance evaluation of a ground-to-train Free Space Optical link communication (G2T FSO) was performed. The system was simulated at $2.5 \mathrm{~Gb} / \mathrm{s}$ link under several weather conditions. Receiver and geometrical loss were included as well. Furthermore, performance was evaluated in terms of received power, Q factor, BER and eye diagram. Substantial vulnerability to severe fog attenuation was found. Although the system was able to operate with acceptable eye height with $\min$ BER of $10^{-38}$.
\end{abstract}

Keywords: Atmospheric attenuation; free space optics; G2T FSO; ground-to-train communications.

\section{Introduction}

Nowadays, Free space optical communication (FSO) has become one of the most prominent technologies. It is on the frontier of next-generation high-speed broadband networks. It uses means of modulated laser beams to transmit data through specific wavelengths, that enables it to deliver extremely high bandwidth with low power and improved security [12]. Besides, it is a tariff-free technology that requires no licensing. It is easy to deploy since it uses half of the RF energy and takes a tenth of an RF antenna diameter [1]. The idea of the use of light as a communication medium is quite old [2]. Although in recent years, advances in optoelectronic component technology and manufacturing offered a massive growth in the field [9]. These advances enabled FSO technology to widen its application spectrum to cover a variety of commercial applications such as Ground to low-earth orbit, unmanned aerial vehicles(UAV's) etc[10]. Currently, it is one of the promising technologies that would address huge bandwidth requirements and last mile bottleneck [11].

On the other hand, there is a demand for reliable, comfortable and fast railway transportation and communication networks. Railway industry relies on communication systems to provide a wide range of solutions to rail operations, safety, security and improved passenger experience [13]. These solutions cover but are not limited to closed circuit television (CCTV), sensors, emergency communications, network supervision and SCADA systems. Furthermore, control based train telecommunication (CBTC) is evolving to simplify systems architecture and improve train remote condition monitoring and diagnostics [3].

Ground-to-train Free Space Optical communication links (G2T FSO), utilizes features of FSO technology to the rail industry. This introduces FSO advantages to the railway industry, which if de- veloped promises higher speed broadband networks, last mile access, and higher speed data rates [15]. By bridging the gap between both technologies, new opportunities to develop intelligent rail transportation systems emerges [14].

In this paper, performance evaluation of a G2T FSO communication link is proposed. This paper is organized as follow: section 2 describes the system design. Section 3 handles G2T FSO required empirical models for channel characterization and simulation setup. Furthermore, simulation results are presented and system performance is analysed in section 4. Finally, conclusions are presented in section 5 .

\section{System Design}

This section consists of two sections. The first section handles the G2T FSO link system model in terms of layout and geometrical properties. Then, a link channel is characterized in terms of fog, rain and geometrical losses.

\subsection{G2T FSO link model}

Figure 1 illustrates a modified model of FSO link for G2T system layout, which comprises of multiple evenly separated ground base stations that are linked to a backbone network [4]. Each base station radiates a Gaussian optical beam that spreads towards roof mounted train transceivers. Base stations are only active when a train passes to conserve energy. Although, PAT systems provide better system stability, coverage, performance and less pointing errors. For the current G2T FSO model, it is assumed that base stations do not have a (PAT) system. 


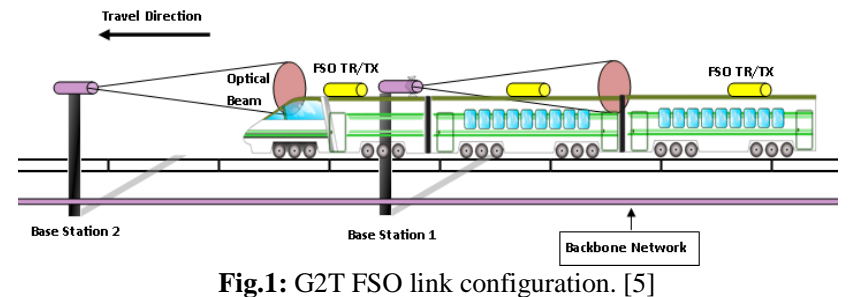

Optical beam shape along the transmission distance and at the receiving terminal are essential criteria in establishing a successful link and evaluating the system performance. Therefore, geometrical properties and are considered as shown in figure 2 , where the train travels along the track distance $L_{\text {Coverage }}$.

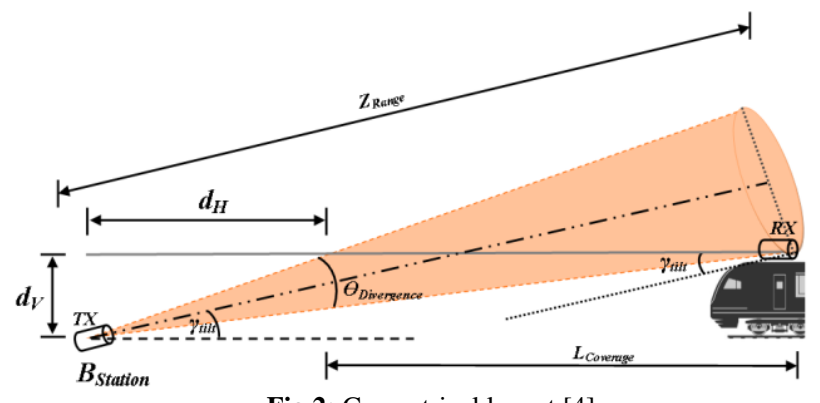

Fig.2: Geometrical layout [4]

Base stations are positioned between horizontal and vertical distances of $d_{H}$ and $d_{V}$ respectively. Since laser beam propagates along $Z_{\text {Range }}$, it spreads and $0.135 \approx 1 / \mathrm{e}^{2}$ of beam peak intensity declines, which leaves $86 \%$ encircled in its width. Thus, high power propagates through $\mathrm{Z}$ axis but sharply drops on the edges of the beam. [4] [2]. In order to formulate the received power of G2T FSO link, it is essential to establish the beam range and divergence. Thus, based on Fig.2 divergence angle $\theta_{\text {Divergence }}$ in (mrad) is defend in equation (1) [4].

$$
\theta_{\text {Divergence }}=\tan ^{-1} \frac{d_{V} L_{\text {Coverage }}}{d_{V}{ }^{2}+d_{H} L_{\text {Coverage }}+\mathrm{d}_{H}{ }^{2}}
$$

Furthermore, by considering tilt angle $\gamma_{\text {Tilt }}$ distance and divergence the effective optical beam axis $\mathrm{Z}$ range is defined as [4]:

$$
Z_{\text {Range }}=L_{\text {Coverage }} \cos \gamma_{\text {Tilt }}+\left(d_{V}{ }^{2}+d_{H}{ }^{2}\right)^{1 / 2} \cos \frac{\theta_{\text {Divergence }}}{2}
$$

Lastly, received power $P_{\text {Received(dB) }}$ of FSO link is defined as [2] :

$$
P_{\text {Re ceived }}=P_{\text {Transmitted }} * \frac{d_{\text {Re ceiver }(m)}{ }^{2}}{\left[d_{\text {Transmitter }(m)}+\left(\theta_{\text {Divergence }} * Z_{\text {Range }[\mathrm{km}]}\right)\right]^{2}} * 10^{\left(-\alpha * \frac{Z_{\text {Range }}}{10}\right)}
$$

Where is $P_{\text {Transmitted }}$ Transmitted power in $(\mathrm{dBm}), Z_{\text {Range }[\mathrm{km}]}$ is the link range in $(\mathrm{km}), \alpha$ is atmospheric attenuation factor $(\mathrm{dB} / \mathrm{km}), d_{\text {Reciver }(m)}$ is the receiver aperture diameter and $d_{\text {, }}$

is the Transmitter aperture diameter. Table 1 demonstrates the geometrical properties of the communication link model.

Table 1: G2T FSO link Geometrical properties.

\begin{tabular}{lcc}
\hline \multicolumn{1}{c}{ Parameter } & Symbol & Value \\
\hline Divergence angle & $\theta_{\text {Divergence }}$ & $0-4.6^{\circ}$ \\
& $\gamma_{\text {Tilt }}$ & $2.25^{\circ}$ \\
LOS tilt angle & $d_{v}$ & $1.435 \mathrm{~m}$ \\
& $d_{H}$ & $15 \mathrm{~m}$ \\
Vertical Base station distance & $L_{\text {Coverage }}$ & $75 \mathrm{~m}$ \\
Horizontal Base station distance & & \\
Track Coverage Distance & & \\
\hline
\end{tabular}

\subsection{Channel Characterization}

Free space optical link utilizes the earth atmosphere as a medium to establish an optical link. This imposes limitations in beam transmission, due to absorption and scattering of photons. Photons interaction with atoms and molecules of air, causes them to disappear or be absorbed [2]. In contrast, if a part of the beam interacts with atoms or molecules during propagation, it causes a redistribution of the beam and it scatters. Absorption, scattering, and extinction are considered as a limiting factor when establishing an FSO link. Using empirical models fog, rain and geometrical losses are described in this section.

\subsubsection{Fog:}

Fog molecules are considered to be major photon absorbers, due to their fine size which is comparable to wavelength transmission windows of FSO. It imposes tremendous effects that lead to limited link rang, which limits the FSO system from achieving high availability. Besides, fog attenuation levels could reach up to [300 $\mathrm{dB} / \mathrm{km}]$. Fog optical attenuation is predicted by using Mie scattering theory. The approach depends on the use of the measure of atmosphere transparency known as Visibility. One of the common models of fog attenuation coefficient is defined in equation (4) [6]:

$\alpha_{F o g[d B / K m]}=\frac{3.91}{V_{k m}}\left(\frac{\lambda_{n m}}{550_{n m}}\right)^{-q}$

Where, $\alpha_{F o g[d B / K m]}$ is the atmospheric attenuation (or cattering) coefficient, $\mathrm{V}(\mathrm{km})$ is visibility, $\lambda_{\mathrm{nm}}$ is the link wavelength and $q$ denotes the scattering size distribution coefficient, according to $\mathrm{KIM}$ it is in equation (5) defined as follow :

$q=\left\{\begin{array}{l}1.6 \\ 1.3 \\ 0.58 V^{\frac{1}{3}}\end{array}\right.$

In contrast, Kruse defines it in equation (6) as:

$q=\left\{\begin{array}{l}1.6 \\ 1.3 \\ 0.16 V+1.34 \\ V-0.5 \\ 0\end{array}\right.$

Based on International visibility code [8], the Table 2 below summarizes attenuation under different fog levels used in G2T FSO link simulation.

Table 2: Fog Attenuation corresponding to various Visibility conditions.

\begin{tabular}{ccc} 
Weather Condition & Visibility Range $(\mathrm{km})$ & Attenuation $(\mathrm{dB} / \mathrm{km})$ \\
\hline Clear Air & 20000 & 0.6 \\
Light Fog & 770 & 18.3 \\
Moderate Fog & 500 & 28.9 \\
Thick Fog & 200 & 75
\end{tabular}

\subsubsection{Rain}

Rain attenuation is given generally by the CARBONNEAU model] as in equation (7) follow [6]:

$\gamma_{\text {Rain }[\mathrm{dB} / \mathrm{Km}]}=k \bullet R_{[\mathrm{mm} / \mathrm{hr}]}^{\alpha}$ 
Where, $k$ and $\alpha$ are constants that depend on predetermined measured values [8]. $R$ rain rate of $\mathrm{R}(\mathrm{mm} / \mathrm{hr})$ and $\gamma_{\text {Rain }}$ is rain attenuation coefficient in $[\mathrm{dB} / \mathrm{km}]$. Various rainfall attenuation for different precipitation rates is tabulated in Table 3.

Table 3: Rain fall Attenuation corresponding to different precipitation types.

\begin{tabular}{ccc}
\hline $\begin{array}{c}\text { Precipitation } \\
\text { Type }\end{array}$ & $\begin{array}{c}\text { Precipitation Rate } \\
(\mathrm{mm} / \mathrm{hr})\end{array}$ & Attenuation $(\mathrm{dB} / \mathrm{km})$ \\
\hline Drizzle & 0.20 & 0.58 \\
Strong & 19.6 & 10.29 \\
Very Strong & 19.34 & 19.34 \\
\hline
\end{tabular}

\subsubsection{Geometrical loss}

The geometrical loss is a function of $\theta_{\text {Divergence(mrad) }}, Z_{\text {Range }[\mathrm{km}]}$ and beam capture area defined as [2]:

$$
\operatorname{Loss}_{\text {Geomertical }}(d B)=10 * \log _{10}\left\{\frac{d_{\text {Re ceiver }(m)}}{d_{\text {Transmitter }(m)}+\left[Z_{\text {Range }(\mathrm{km})} * \theta_{\text {Divergence(mrad) }}\right.}\right\}^{2}
$$

Where $\mathrm{d}_{\text {Receiver }(m)}$ is the receiver aperture diameter, $d_{\text {Transmitter }(\mathrm{m})}$ is the transmitter aperture diameter, $Z_{\text {Range }[\mathrm{km}]}$ is the line-of-sight range in $\mathrm{km}$ and Loss $_{\text {Geometrical }(d B)}$ is the specific geometrical loss in disciples.

\section{Simulation:}

In this paper, simulations have been carried out over two stages The first stage comprised of numerical computation of geometrical properties and channel characteristics. It included link range, beam divergence, fog, rain and geometrical losses. After that, G2T FSO communication system with OOK-NRZ modulation has been constructed and analyzed under seven scenarios each with different weather conditions. The simulation block diagram is shown in Figure 3.
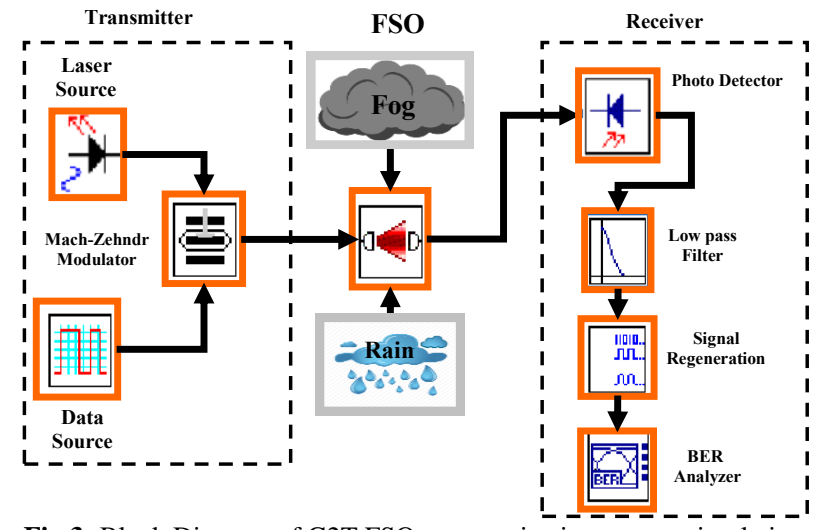

Fig.3: Block Diagram of G2T FSO communication system simulation.

G2T FSO communication links consist of three major blocks Transmitter, FSO channel, and receiver. The transmitter block has an optical source (laser diode), electrical signal NRZ data source and an external modulator. Transmitter block converts electrical binary data and optical laser beam into a pulsed modulated signal that probates through FSO channel. Moreover, FSO channel consists of transmitter and receiver telescope. In addition, to atmospheric noises in $[\mathrm{dB} / \mathrm{km}]$, that are fed to the channel to evaluate its performance. Lastly, the receiver block detects, amplify and regenerate the optical signal to be converted into digital binary. It uses an Avalanche photodetector, low pass filter and signals regeneration blocks. BER analyzer tool is used to visuals, evaluate and analyze the received signal. The simulation has been carried out at a wavelength of $850 \mathrm{~nm}$, due to its transmission window, robustness to scattering, inexpensive components, and high performance. Simulation parameters are given in the table below.

Table 4: System parameters utilized in G2T FSO link analysis

\begin{tabular}{ccc}
\multicolumn{3}{c}{ Table 4: System parameters utilized in G2T FSO link analysis } \\
\hline Parameter & Symbol & Value \\
\hline Laser Wavelength & $\lambda$ & $850 \mathrm{~nm}$ \\
Bit rate & $B_{r}$ & $2.5 \mathrm{Gbps}$ \\
Transmitter Optical power & $P_{\text {Transmitted }}$ & $15 \mathrm{~mW}$ \\
Transmitter aperture diameter & $d_{\text {Receiver }}$ & $7 \mathrm{~mm}$ \\
Receiver aperture diameter & $d_{\text {Transmitter }}$ & $5 \mathrm{~mm}$ \\
APD Responsitivity & $\Re$ & $0.59 \mathrm{~A} / \mathrm{W}$ \\
Receiver Gain & $G_{\text {Receiver }}$ & $24 \mathrm{~dB}$ \\
Dark Current & $i_{\text {dark }}$ & $10 \mathrm{nA}$ \\
Geometrical loss & Loss $_{\text {Geometrical(dB) }}$ & $3.366 \mathrm{~dB}$ \\
\hline
\end{tabular}

\section{Results and discussion}

\subsection{Numerical results}

In this section, numerical analysis of equations (1), (2) and (8) is performed, to evaluate a variety of geometrical properties of G2T FSO link as shown in Fig. 4 (a), (b) and (c).

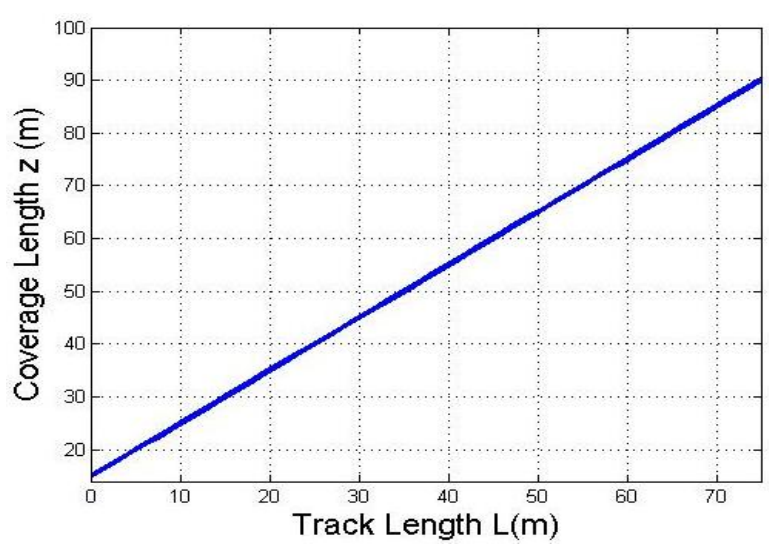

(a)

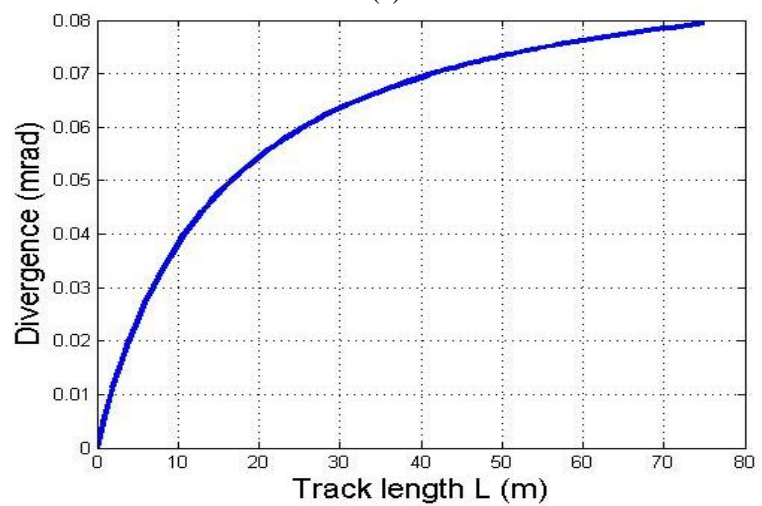

(b)

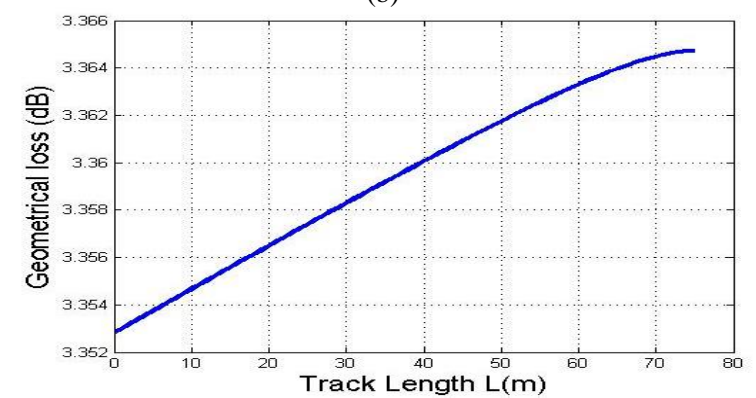

(c)

Fig.4: (a) $\mathrm{L}_{\text {Coverage }}$ versus ZRange. (b) $\mathrm{L}_{\text {Coverage }}$ versus $\theta_{\text {divergence }}$ (c) L versus. Loss $_{\text {Geometrical } d B}$

By comparing figures 4 (a) and (b) it is noted that the relation between track length, coverage and divergence angle is directly proportional. Therefore, the further the train from the base station 
the higher geometrical losses are. Additionally, the link range between the base station and the locomotive stretches beyond the span of the track by $15 \mathrm{~m}$. Therefore, by substituting the results from equation (1) and (2) into equation (8), Loss Geomerrical (dB) $_{\text {along }}$ the track is calculated and shown in figure 4 (c). Geometrical losses and coverage range are inversely proportional. Moreover, since coverage range, divergence angles and geometrical losses are key factors in G2T FSO link deployment. They were incorporated in the link design and simulation.

\subsection{Simulation results}

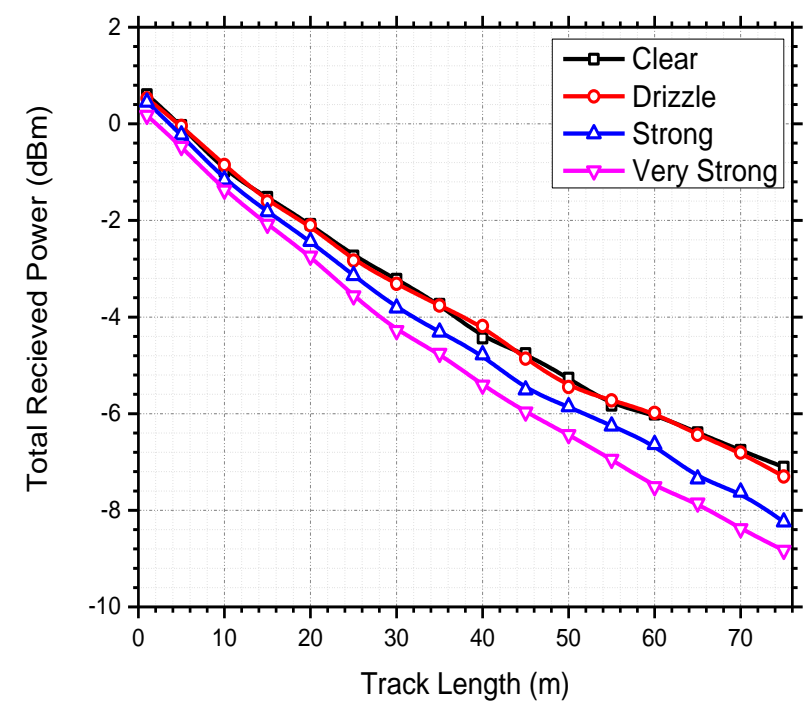

(a)

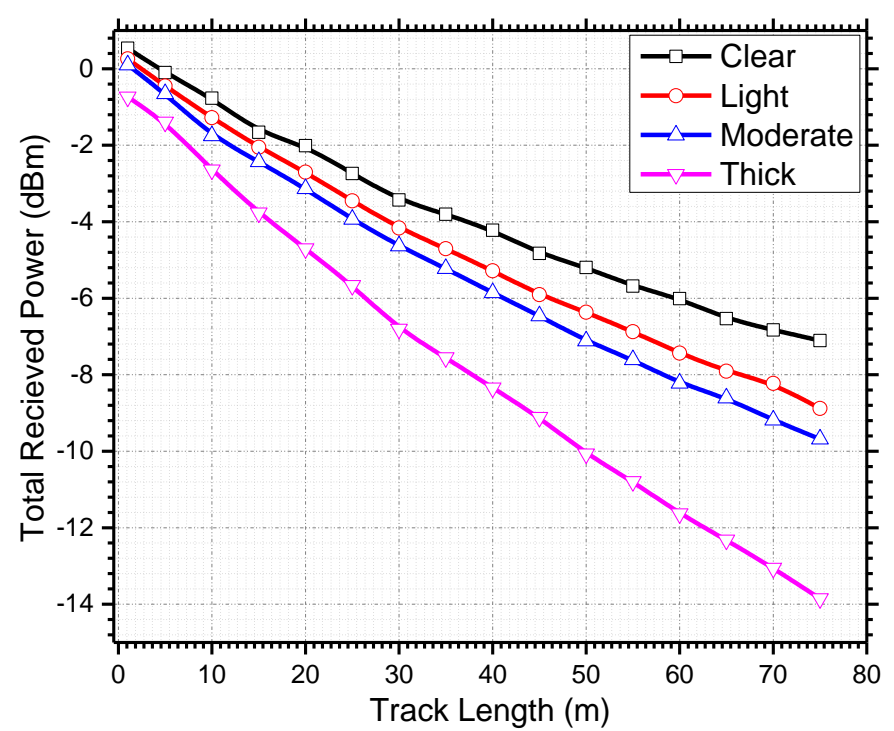

(b)

Fig.5: Received power versus (a) Rain. (b) Fog Attenuation.

Figure 5 (a) illustrates variations in simulated total received power along track length under different levels of rain and fog attenuation. Firstly, power levels slightly drop under drizzle rain rate when compared to clear weather conditions. However, under strong and very strong rain rates received energy drops by $1.13 \mathrm{dBm}$ and $1.72 \mathrm{dBm}$ respectively. That is an average decrease of $22.7 \%$ and $38.8 \%$ for cases higher than a light drizzle. On the other hand, it is clear that fog attenuation has a higher impact on FSO communication link. Figure 5 (b) shows a sharp decrease under thick fog condition, which impairs visibility and causes a $3.81 \mathrm{dBm}$ or $84.14 \%$ power drop along coverage distance. Besides, light and moderate haze degrade received signal by an average fall of $0.91 \mathrm{dBm}$ and $1.45 \mathrm{dBm}$ respectively.
Another way to evaluate link performance is to examine the Q factor of the system. It is widely used to characterize the quality of received signals. It indirectly points to BER and provides indicators of bit-error deterioration within FSO link. Figure 6 instates the simulated drop of $\mathrm{Q}$ factor versus distance under various intensities fog and rain attenuations. It is noted that thick fog sharply deteriorates link quality down to 23.6 factors at $75 \mathrm{~m}$, whereby the system performs with higher $\mathrm{Q}$ factor under stronger rain rates at a similar distance. In contrast, the signal quality is significantly higher under rain attenuations, when compared to different fog disturbances levels.

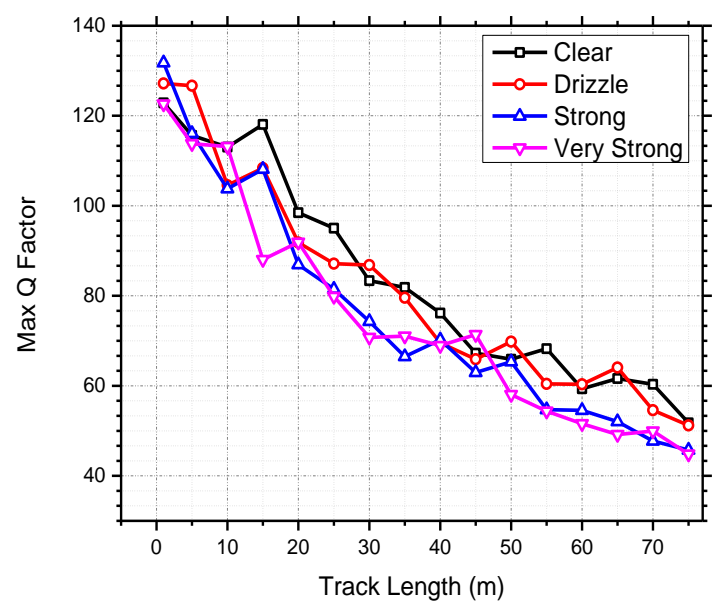

(a)

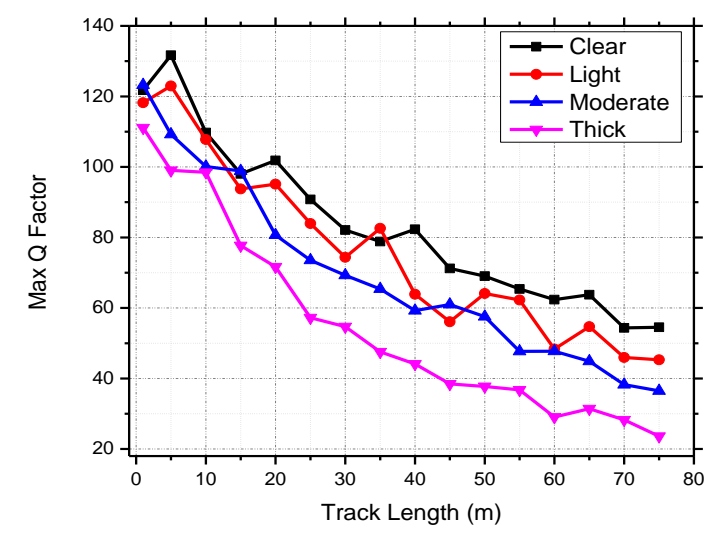

(b)

Fig.6 : Q factor versus (a) Rain. (b) Fog Attenuation.

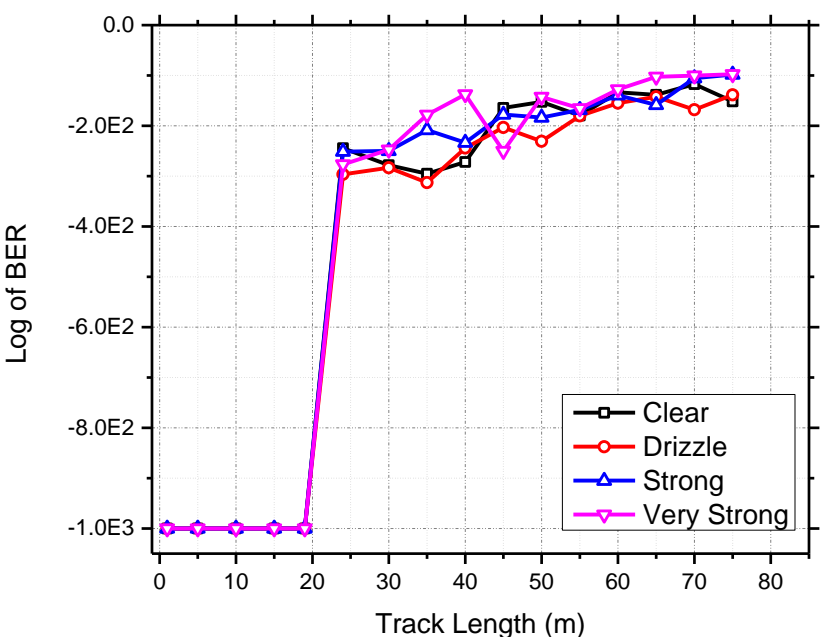

Fig. 7: $\log$ of BER under Rain attenuation . 


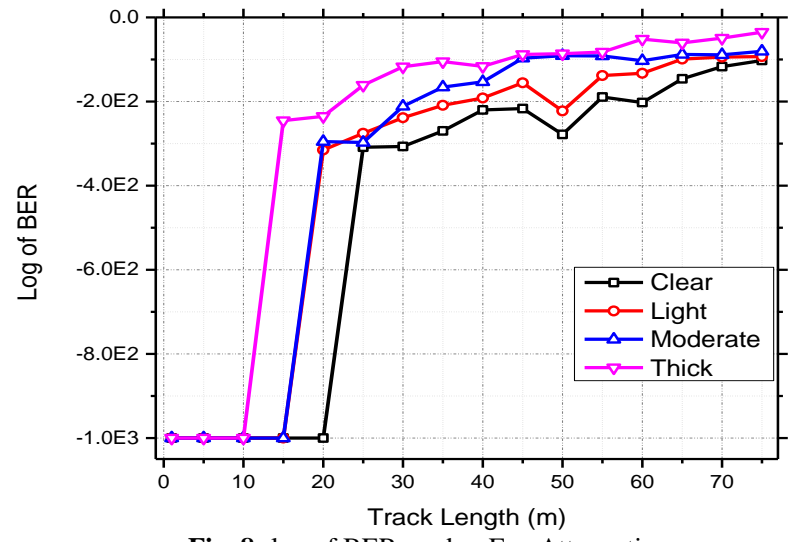

Fig. 8: $\log$ of BER under Fog Attenuation.

Bit Error rate indicates the probability of false detection of bits by decision block of a receiver. In general, a BER of less than $10^{-9}$ is desirable, which reflect a possibility of one-bit error in a thousand million. The $\log$ of bit-error-rate along $\mathrm{L}$ for various weather conditions is shown in figure 7 and figure 8. Under rain attenuation, BER sharply shifts after $20 \mathrm{~m}$ travel along the rail then gradually increase with distance. Furthermore, under moderate and light haze levels the rate of error changes at $15 \mathrm{~m}$. However, under lower visibility, this change occurs $10 \mathrm{~m}$ prior to clear weather conditions. Lastly, a minimum BER of $5.9 \times 10^{-98}$ and $5.81 \times 10^{-36}$ are feasible despite atmospheric and geometrical disturbances, due to the short-range nature of G2T FSO link.

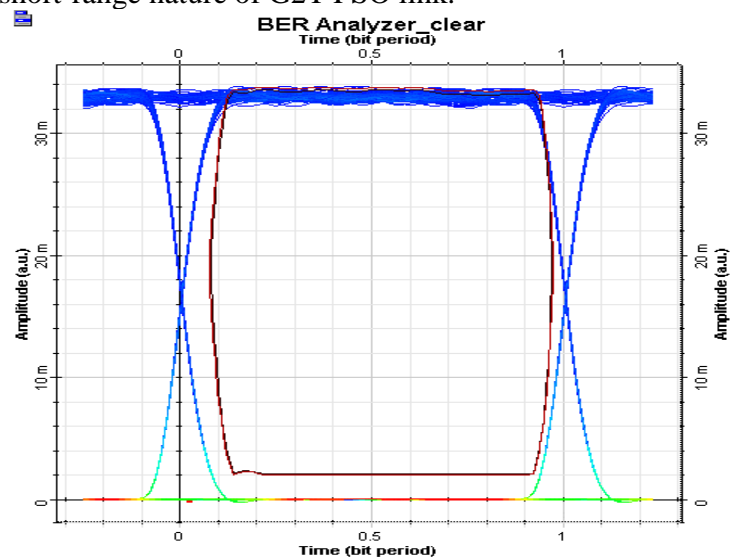

Fig. 9: Eye diagram of an Ideal weather condition.

Figure 9 and Figure 10 (a) and (b) demonstrate the eye diagrams at a $75 \mathrm{~m}$ distance under clear, very strong and thick fog conditions. Acceptable eye-opening is present and sufficient to detect the received optical signal. By analyzing the shape of each case, effects of atmospheric attenuation disturbances are clear. Besides, under very strong and thick fog condones, eye height considerably decreases by $24.78 \%$ and $83.13 \%$ respectively.

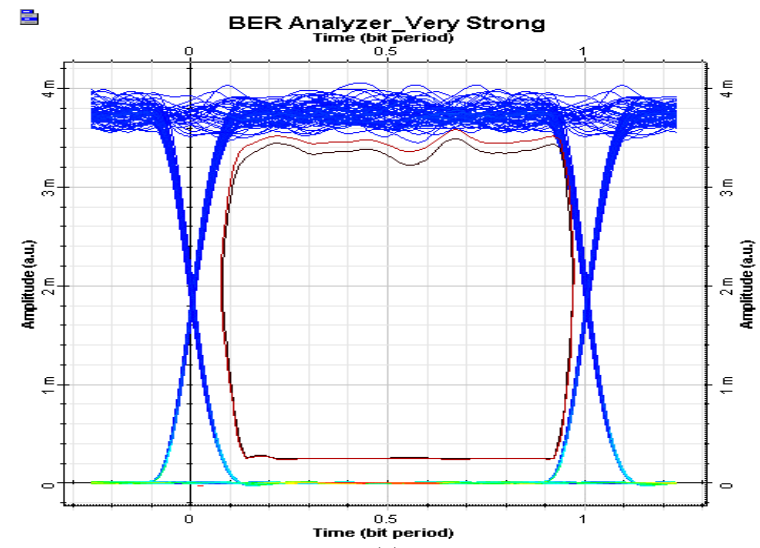

(a)

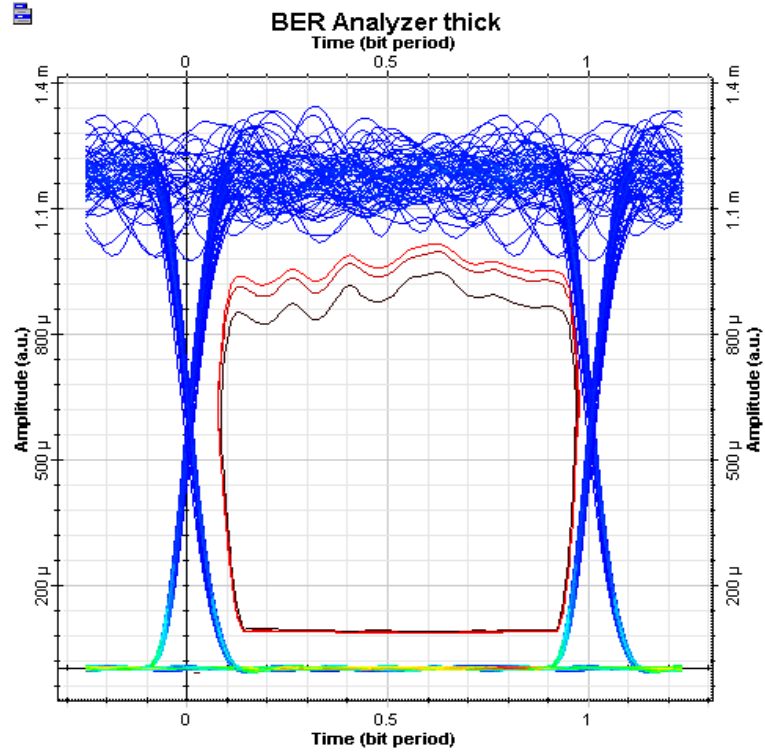

(b)

Fig.10: Eye diagrams of (a) Very strong Rain attenuation. (b)Thick Fog attenuation.

\section{Conclusion}

Although there are many advantages of FSO technology, severe weather effects raise concerns about sustainable system availability and performance. From simulation results, it is observed that a substantial drop of $84 \%$ in total received power under thick fog condition. In contrast to a $38.8 \%$ deterioration under heavy rain. Even though, FSO link maintained an acceptable operating eye height over $2.5 \mathrm{Gbps}$ with minimum BER of $5.81 \times 10^{-36}$. An essential criterion of a communication system is the high availability of 99.9\%. Moreover, drops in BER and Q factor performances over distances of $10 \mathrm{~m}$ and $20 \mathrm{~m}$ for fog and rain conditions are also noted. Further improvements and implementation of mitigations techniques are undergoing to improve the link range and sensitivity to severe atmospheric conditions.

\section{Acknowledgement}

The authors gratefully acknowledge the Faculty of Electrical and Electronic Engineering (FKEE) and Centre of Graduate Studies (PS) for their support.

\section{References}

[1] Kaushal H, Jain VK \& Kar S (2017) Free space optical communication Springer India;1-11.

[2] Bloom S, Korevaar E, Schuster J \& Willebrand H (2003), Understanding the performance of free-space optics. Journal of optical Networking 2(6) 178.

[3] Qureshi KN \& Abdullah AH (2003), A survey on intelligent transportation systems. Middle-East Journal of Scientific Research. 15(5) 629-42.

[4] Paudel R, Poliak J, Ghassemlooy Z, Wilfert O \& Leitgeb E (2014) Apr. curved track analysis of fso link for ground-to-train communications. Radioengineering 1;23(1) 452-9.

[5] Mabrouk WA \& Abdullah MF (2017), fso g2t communications in tropical climate: an overview. AIP Conference Proceedings Vol. 1883, No. 1, p. 020009.

[6] Naboulsi M, Sizun H \& Fornel F (2005), Propagation of optical and infrared waves in the atmosphere. Proceedings of the union radio scientifique international.

[7] Kim II, McArthur B \& Korevaar EJ (2001), Comparison of laser beam propagation at $785 \mathrm{~nm}$ and $1550 \mathrm{~nm}$ in fog and haze for optical wireless communications. InOptical Wireless International So- 
ciety for Optics and Photonics Communications III Vol. 4214, pp. 26-38.

[8] Recommendation IT. prediction methods required for the design of terrestrial free-space optical links. International telecommunication Union. 2007:1814

[9] Forrest SR. Monolithic optoelectronic integration: A new component technology for lightwave communications. IEEE Transactions on Electron Devices. 1985 Dec;32(12):2640-55

[10] Khalighi MA, Uysal M (2014), Survey on free space optical communication: A communication theory perspective. IEEE communications surveys \& tutorials. 16(4):2231-58.

[11] Chan VW. (2006), Free-space optical communications. Journal of Lightwave technology 1;24(12):4750-62.

[12] Kim II, Korevaar EJ (2001). Availability of free-space optics (FSO) and hybrid FSO/RF systems. InOptical Wireless Communications IV 2001 Nov 27 (Vol. 4530, pp. 84-96). International Society for Optics and Photonics.

[13] Shafiullah GM, Gyasi-Agyei A, Wolfs P. Survey of wireless communications applications in the railway industry. InWireless Broadband and Ultra Wideband Communications, 2007. AusWireless 2007. The 2nd International Conference on 2007 Aug 27 pp. 65-65 IEEE.

[14] Ai B, Cheng X, Kürner T, Zhong ZD, Guan K, He RS, Xiong L, Matolak DW, Michelson DG, Briso-Rodriguez C. Challenges toward wireless communications for high-speed railway. IEEE transactions on intelligent transportation systems (5):2143-58.

[15] Ai B, Guan K, Rupp M, Kurner T, Cheng X, Yin XF, Wang Q, Ma GY, Li Y, Xiong L, Ding JW (2015). Future railway servicesoriented mobile communications network. IEEE Communications Magazine 53(10):78-85. 